\title{
Review Article \\ The Role of TL1A and DR3 in Autoimmune and Inflammatory Diseases
}

\author{
Yoshihiro Aiba ${ }^{1}$ and Minoru Nakamura ${ }^{1,2,3}$ \\ ${ }^{1}$ Clinical Research Center, National Hospital Organization Nagasaki Medical Center, Kubara 2-1001-1, Omura 856-8562, Japan \\ ${ }^{2}$ Department of Hepatology, Nagasaki University Graduate School of Biomedical Sciences, Kubara 2-1001-1, Omura 856-8562, Japan \\ ${ }^{3}$ Headquarters of PBC Research in NHOSLJ, Clinical Research Center, National Hospital Organization Nagasaki Medical Center, \\ Kubara 2-1001-1, Omura 856-8562, Japan \\ Correspondence should be addressed to Minoru Nakamura; nakamuram@nmc.hosp.go.jp
}

Received 31 May 2013; Accepted 2 December 2013

Academic Editor: Linda Burkly

Copyright ( 2013 Y. Aiba and M. Nakamura. This is an open access article distributed under the Creative Commons Attribution License, which permits unrestricted use, distribution, and reproduction in any medium, provided the original work is properly cited.

TNF-like ligand 1A (TL1A), which binds its cognate receptor DR3 and the decoy receptor DcR3, is an identified member of the TNF superfamily. TL1A exerts pleiotropic effects on cell proliferation, activation, and differentiation of immune cells, including helper T cells and regulatory T cells. TL1A and its two receptors expression is increased in both serum and inflamed tissues in autoimmune diseases such as inflammatory bowel disease (IBD), rheumatoid arthritis (RA), and ankylosing spondylitis (AS). Polymorphisms of the TNFSF15 gene that encodes TL1A are associated with the pathogenesis of irritable bowel syndrome, leprosy, and autoimmune diseases, including IBD, AS, and primary biliary cirrhosis (PBC). In mice, blocking of TL1A-DR3 interaction by either antagonistic antibodies or deletion of the DR3 gene attenuates the severity of multiple autoimmune diseases, whereas sustained TL1A expression on T cells or dendritic cells induces IL-13-dependent small intestinal inflammation. This suggests that modulation of TL1A-DR3 interaction may be a potential therapeutic target in several autoimmune diseases, including IBD, RA, AS, and PBC.

\section{Characteristics of TL1A and DR3}

1.1. TL1A. TL1A, also referred to as vascular endothelial growth inhibitor (VEGI)-251, is a member of the tumor necrosis factor superfamily (TNFSF) of ligands, which was identified by Migone et al. in 2002 [1]. Although TL1A was identified as a longer variant of TL1/VEGI, the fourth exon of TL1A encodes the majority of TL1/VEGI, and it has been presumed that the original TL1/VEGI was a cloning artifact. TL1A exhibits approximately $20-30 \%$ homology to other TNFSF members [1]. Human TL1A consists of 251 amino acids: 35 in the cytoplasmic domain, 24 in the transmembrane region, and 192 in the extracellular domain. There are two potential N-linked glycosylation sites in the TL1A amino acid sequence, specifically Asn residues at amino acids 133 and 229 [1]. TL1A is a type II transmembrane protein. TL1A is initially expressed as a membrane-bound protein and is subsequently released as a soluble protein via ectodomain shedding by a metalloproteinase such as TNF- $\alpha$ converting enzyme (TACE) $[2,3]$. TL1A expression is detected on human umbilical vein endothelial cells and synovial fibroblast-like cells and is upregulated by stimulation with proinflammatory cytokines such as TNF- $\alpha$, IL-1, and PMA, a phorbol ester known to be a potent activator of protein kinase C $[1,4]$. TL1A expression has also been confirmed on antigen-presenting cells and lymphocytes that are activated by Toll-like receptor (TLR) ligands, enteric bacteria, and $\mathrm{F} c \gamma$ receptor $(\mathrm{Fc} \gamma \mathrm{R})$ crosslinking [5-7].

1.2. DR3. DR3, also known as APO-3, TRAMP, LARD, and WSL-1, is a member of the tumor necrosis factor receptor superfamily (TNFRSF) with a typical death domain that consists of an approximately 60-amino-acid globular bundle of 6 conserved $\alpha$ helices found in the cytoplasmic region. Although DR3 is most homologous to TNFR1, which is 
widely expressed, its expression is mostly restricted to lymphocytes such as NK cells and T cells, in particular NKT cells and is enhanced upon their activation [8-10]. DR3 is more highly expressed on Th17 cells than on Th1 and Th 2 cells, and is also expressed on naturally occurring and TGF- $\beta$-induced Treg cells (n-Treg and i-Treg, resp.) [11-13]. It was recently shown that DR3 expression on B cells was induced by antiIgM stimulation, although its expression was not detectable on resting B cells [14]. There are several expression differences between human and mouse [9]. DR3 splicing variants of 13 in human $[8,15,16]$ and 3 in mice [17] have been identified. Pappu et al. showed that DR3 splicing variants are differentially expressed on T-cell subsets in mice [13].

\section{Role of TL1A-DR3 Signaling in Cell Fate Determination}

TL1A-DR3 signaling induces both NF- $\kappa$ B activation and apoptosis in vitro $[1,18,19]$. TL1A-DR3 interaction induces the formation of signaling complexes containing TRADD, TRAF2, and RIP and activates the NF- $\kappa$ B and MAPK pathways (ERK, p38, and JNK). The activation of NF- $\kappa$ B induces c-IAPs, which protect against apoptosis [20]. On the other hand, DR3 overexpression in embryonic cells also induces FADD- and caspase-8-dependent apoptosis [21, 22]. Blocking of TL1A-DR3 signaling by adding NF- $\kappa$ B inhibitors or protein synthesis inhibitors induces apoptosis [20], suggesting that NF- $\kappa$ B activation by TL1A-DR3 interaction is responsible for resistance to apoptosis. Analysis of DR3-deficient mice has shown that DR3 is required for negative selection in the thymus [23]. Inhibition of TL1A-DR3 interaction has shown that TL1A-DR3 signaling is required for effective T-cell immune responses in the target organs of T-cell-mediated autoimmune diseases and inflammatory diseases [24]. Thus, TL1ADR3 signaling may be involved in lymphocyte homeostasis by modulating either cell death or lymphocyte activation.

\section{Role of TL1A and DR3 Signaling in the Immune System}

3.1. Th1. Under TCR stimulation, TL1A induces cell proliferation and the secretion of proinflammatory cytokines (including IFN- $\gamma$, GM-CSF, and TNF- $\alpha$ ) in T cells, in particular memory $\mathrm{CD}^{+} \mathrm{T}$ cells $[1,25]$. Sustained TL1A expression on $\mathrm{T}$ cells or dendritic cells leads to an increase in the number of activated $\mathrm{CD} 4^{+} \mathrm{T}$ cells and memory $\mathrm{CD} 4^{+} \mathrm{T}$ cells, whereas sustained TL1A expression on dendritic cells does not stimulate conventional $\mathrm{T}$ cells in the absence of TCR stimulation in vivo, suggesting that TL1A may act as a costimulator for $\mathrm{T}$ cells to regulate inflammatory cytokines and cell proliferation $[26,27]$. TL1A synergizes with IL$12 /$ IL-18 to promote IFN- $\gamma$ production in $\mathrm{T}$ cells in an antigen-independent manner $[25,28]$. TL1A itself cannot directly induce Th1 differentiation of native $\mathrm{CD}^{+} \mathrm{T}$ cells, while TL1A-deficient mice show the decrease of IFN- $\gamma$ producing $\mathrm{CD}^{+} \mathrm{T}$ cells [13]. Collectively, it is speculated that TL1A indirectly or synergistically with other cytokines enhances Thl responses of activated and memory $\mathrm{CD}^{+} \mathrm{T}$ cells. The differential T-cell responsiveness for TL1A between naïve and activated/memory $\mathrm{T}$ cells might be explained by the regulation of DR3 splicing variants, in particular fulllength transmembrane variant, which encodes complete transmembrane DR3 protein. The expression of full-length DR3 mRNA and protein is low level or not detected in naïve and resting T cells [8], whereas it is upregulated in activated $\mathrm{T}$ cells $[9,25,29]$. In mice with chronic intestinal inflammation, the transmembrane DR3 expression is upregulated [25].

3.2. Th17. Although exogenous TL1A induces IL-2 secretion and responsiveness in T cells $[1,24], \mathrm{IL}-2$ is a negative regulator for Th17 cells [30]. Therefore, when IL-2 is blocked, exogenous TL1A induces the differentiation of Th17 cells from naïve $\mathrm{CD}^{+}{ }^{+} \mathrm{T}$ cells stimulated with TCR under Th17 polarization condition in vitro. TL1A also induces the proliferation of invitro-differentiated Th17 effector cells but neither Th1 nor Treg cells even in the absence of TCR stimulation $[11,13]$. This differential TL1A responsiveness in T-cell subset might be explained by DR3 expression. DR3 expression is upregulated at a later stage but not early stage of Th17 differentiation. Total DR3 expression is increased in Th17 cells as compared with Th1 and Th2 cells, and full-length transmembrane DR3 expression is increased in Th17 cells as compared with Treg cells [13]. Thus, TL1A-DR3 interaction might preferentially act on Th17 cells and differentially affect the differentiation and maturation of Th17 cells. On the other hand, TL1A inhibits Th17 cell differentiation even in the presence of antiIL-2 neutralizing antibody in vitro [11]. Activation of STAT1 signaling is induced by inflammatory cytokines such as IL27, IFN- $\gamma$, and type I IFN and inhibits Th17 differentiation in vitro [31]. However, the inhibitory mechanism of Th17 differentiation by TL1A was independent of activation of STAT1 signaling as well as IL-2 signaling [11]. Further, DR3 is dispensable for Th1, Th2, and Th17 differentiation from naïve CD4+ $\mathrm{T}$ cells in vitro [24]. Thus, the role of TL1A in Th17 differentiation is still controversial in vitro. However, TL1A transgenic mice [26, 27], and Th17-mediated autoimmune disease model mice, experimental autoimmune encephalomyelitis [13] and dextran-sulfate-induced chronic colitis [32], show that TL1A-DR3 interaction could positively regulate Th17 cell function in vivo. Further research will be required to elucidate the regulatory mechanism of TL1A-DR3 interaction for Th17 cell function in vitro and in vivo.

3.3. Th2. TL1A-DR3 interaction is involved in Th2- as well as Th1- and Th17-mediated immune responses. Transgenic mice that constitutively express TL1A specifically in T cells or dendritic cells develop Th2 cytokine IL-13-dependent small intestinal inflammation $[26,27]$. Intranasal immunization with ovalbumin (OVA) together with TL1A in mice induces Th2-mediated immune responses, including OVA-specific IgG1 antibody production in serum, IgA antibody production in mucosal tissues, and the production of Th2 cytokines IL4 and IL-5 from OVA-restimulated splenocytes in vitro [33]. Studies have shown that in DR3-deficient mice, or following blockade of TL1A-DR3 interaction by TL1A neutralization antibodies, OVA-induced lung inflammation is attenuated 
and Th 2 cytokines IL- $4,-5$, and -13 production is reduced in a mouse model of asthma $[10,24]$. In mice with small intestinal inflammation or OVA-induced lung inflammation, NKT cells, activated and memory $\mathrm{CD}^{+} \mathrm{T}$ cells, or eosinophils are likely to be a main source of the Th2 cytokines that are induced by the TL1A-DR3 signaling pathway [10, 24, 26, 27], suggesting that TL1A-DR3 signaling in these cells might be a therapeutic target in asthma and ulcerative colitis.

3.4. Treg Cells. TL1A transgenic mice show the proliferation and activation of Treg cells in the secondary lymphoid organs and the small intestinal lamina propria [26, 27, 34]. Although exogenous TL1A itself does not affect either n-Treg or i-Treg proliferation in vitro, it promotes Treg cell proliferation in the presence of antigen presenting cells with TCR stimulation both in vitro and in vivo $[13,35]$, suggesting that TCR signaling is required for costimulation of Treg cells as well as conventional T cells by TL1A. Agonistic anti-DR3 antibodies expand the proliferation of preexisting Treg cells in a manner dependent on TCR and IL-2 signaling in vivo, and the expanded Treg cells inhibit OVA-induced lung inflammation [12]. Although Treg cells derived from TL1A-treated mice have highly suppressive activity ex vivo, both exogenous TL1A and agonistic anti-DR3 antibodies directly inhibit the suppressive activity of Treg cells in vitro $[12,35]$. Treg cells derived from mice that constitutively express TL1A under the CD11 promoter attenuate the ability to suppress conventional T cells in vitro [26], whereas Treg cells derived from mice constitutively expressing TL1A under the CD2 promoter maintain their suppressive ability [27]. These results suggest that the effect of TL1A-DR3 interaction on T cells might be highly dependent on experimental conditions in vitro or the context of the immune response that is being modulated in vivo.

3.5. NK and NKT Cells. TL1A and agonistic anti-DR3 antibodies synergize with IL-12 and IL-18 to augment IFN- $\gamma$ production in NK cells and NKT cells as well as T cells [28]. The fold-induction of IFN- $\gamma$ production by the addition of TL1A is significantly lower in NK cells and NKT cells than in $\mathrm{CD}^{+} \mathrm{T}$ cells and $\mathrm{CD}^{+} \mathrm{T}$ cells. The combination of IL-12 and IL-18 drastically increased the DR3 expression in NK cells but minimally in T cells. These data suggest that the augmentation of IL-12/IL-18-induced IFN- $\gamma$ in response to TL1A is differentially induced in T cells and NK cells. TL1A also enhances IL-12/IL-18-induced NK cell cytolytic activity, which is independent of IFN- $\gamma$ production [36], suggesting that TL1A might be an attractive molecule for tumor therapy. Agonistic anti-DR3 antibodies costimulate the proliferation and IL-13 production of NKT cells stimulated with $\alpha$-galactoceramide or anti-CD3 antibodies [10]. NKT-deficient mice, which are resistant to OVA-induced allergic lung inflammation, restore the lung inflammation upon adaptive transfer of wild-type NKT cells, but not after transfer of dominant negative DR3 transgenic NKT cells, suggesting that DR3 signals in NKT cells play an important role for triggering lung inflammation [10].
3.6. $B$ Cells. In contrast to T cells, there have been few reports on the significance of TL1A-DR3 interaction in B cells. Membrane-bound TL1A expression on resting B cells was found to be at very low levels in mice [27]. TL1A expression is not induced in B cells either during resting or activated conditions. In vitro, TL1A directly reduces B-cell proliferation induced by anti-IgM antibodies and IL-2, whereas it does not affect B-cell proliferation induced by a combination of antiIgM antibodies and other B-cell-specific stimulators, namely, CpG oligodeoxynucleotide and CD40 ligand [14].

Collectively, these reports indicate that TL1A-DR3 interaction exerts pleiotropic effects on adaptive immune cells, including their activation, proliferation, differentiation, cytokine production, and maintenance.

\section{Association of TNFSF15 Gene Polymorphisms with Autoimmune and Inflammatory Diseases}

To examine the association of TNFSF15 gene polymorphisms with autoimmune diseases, Yamazaki et al. performed a genomewide case-control study and found that TNFSF15 gene polymorphisms are associated with the susceptibility to $\mathrm{CD}$ in a Japanese population as well as IBD in a European population [37]. Subsequent replication studies and genomewide association studies have revealed that TNFSF15 is only one gene that is associated with CD or IBD in both Asian and Caucasian population [38-45]. TNFSF15 gene polymorphisms are also associated with the severity of CD and IBD in Japanese and Caucasian population, respectively [46-48]. TNFSF15 haplotypes A and B (which consist of five polymorphisms: rs3810936, rs6478108, rs6478109, rs7848647, and rs7869487) are risk and protective factors, respectively, for susceptibility in both Asian CD and Caucasian IBD patients $[37,39,49]$, and haplotype $B$ is a risk factor for severity and antibody status for E. coli outer membrane porin $\mathrm{C}$ in Jewish CD patients $[49,50]$. A polymorphism of TNFSF15 haplotype $\mathrm{A}$ increases promoter activity in stimulated $\mathrm{T}$ cells [51], whereas TNFSF15 haplotype B is associated with increased soluble and membrane TL1A expression in some Jewish CD patients [50]. In addition to IBD, TNFSF15 gene polymorphism rs4263839 is associated with susceptibility to irritable bowel syndrome and ankylosing spondylitis in Caucasians $[52,53]$, and we recently found that TNFSF15 gene polymorphism rs4979462 is associated with susceptibility to PBC in a Japanese population [54]. These findings suggest that TNFSF15 gene polymorphisms contribute to altered TL1A production, leading to the pathogenesis of autoimmune and inflammatory diseases. In addition to TNFSF15, polymorphisms of $I L-23 R$ and $I L-12 A / I L-12 R B 2$ are associated with susceptibility to IBD $[55,56]$ and PBC [57], respectively. TL1A and IL-23 or IL-12 synergistically induce Th1- and Th17effector cells, implicating the TL1A-IL12/IL-23 pathway in the pathogenesis of both IBD and PBC. Zhang et al. reported that TNFSF15, NOD, and $I L-23 R$ are susceptibility genes for leprosy in a Chinese population $[58,59]$. These genes are also susceptibility genes in $\mathrm{CD}$, suggesting that $\mathrm{CD}$ and leprosy 
may share a common disease pathway, in particular innate immunity and inflammatory responses.

\section{Role of TL1A and DR3 in Autoimmune and Inflammatory Diseases}

5.1. $R A$. TL1A expression is elevated in the serum, synovial fluid, and synovial tissues of RA patients, in particular patients who are positive for rheumatoid factor (RF). Its expression is correlated with the severity of RA $[6,60]$. DR3 gene duplication is more prevalent in RA patients as compared to healthy subjects [61]. Immunohistochemical staining found that TL1A-positive cells in the synovial tissue of RA patients, in particular RF-positive patients, are positive for CD14 and CD68, which are surface markers of macrophages and monocytes [6]. TL1A is induced in human synovial fibroblasts stimulated with TNF- $\alpha$ and IL-1 $\beta$ [4] and in monocytes stimulated with insoluble immune complexes derived from RA patients [6]. TL1A induces T cells to secrete TNF- $\alpha$ and IL-17 under TCR stimulation or Th17 polarization conditions, respectively [4], and it synergizes with IFN- $\gamma$ and augments the production of CXCL8 and matrix metalloproteinase 9 in the human monocytic cell line THP1 [62]. These inflammatory cytokines and chemokines are associated with RA pathology, and therefore it is possible that TL1A and these inflammatory cytokines form a vicious loop that aggravates RA pathogenesis. Indeed, administration of the anti-TNF- $\alpha$ monoclonal antibody adalimumab decreases serum TL1A levels in RA patients [60]. In a mouse model, TL1A administration exacerbated collagen-induced arthritis (CIA) and increased the germinal center formation of spleen and serum anticollagen autoantibodies that are pathogenic in RA [4]. DR3 knockout mice show resistance to development of adverse bone pathology in experimental antigen-induced arthritis (AIA), and TL1A promotes osteoclastogenesis in a DR3-dependent manner in AIA model mice [63], suggesting that DR3 is involved in the generation of osteoclasts at site of bone pathology. Collectively, TL1A-DR3 interaction forms a part of the inflammatory cytokine network and contributes to RA pathogenesis by promoting osteoclastogenesis and the production of inflammatory cytokines and autoantibodies. Furthermore, administration of neutralizing antibodies against TL1A ameliorated both AIA and CIA [63], suggesting that the TL1A-DR3 pathway may be a potential therapeutic target in RA patients.

There has been growing evidence that RA patients are at elevated risk for cardiovascular disease [64]. It was reported that TL1A and its receptors are correlated with atherosclerosis [65]. TL1A regulates the expression of genes implicated in the uptake (scavenger receptors such as SR-A, SR-B1, and CD36) and efflux of cholesterol (ABCG-1, ABCA-1, and ApoE), leading to promotion of foam cell formation in human macrophage [65]. This TL1A-induced macrophage foam cell formation is dependent on DR3. DR3 itself also promotes macrophage foam cell formation by regulating the expression of genes implicated in the uptake and efflux of cholesterol [65]. In addition, it was recently reported that elevated serum TL1A at baseline positively correlates with the progression of atheromatic plaque height in RA patients [66]. A combination of low TL1A and undetectable DcR3 levels in serum at the baseline correlates with decrease of new atheromatic plaques in carotid arteries and/or femoral arteries of RA patients over a follow-up period of 3.5 years, suggesting that serum TL1A and DcR3 levels might predict a preserved atherosclerosis profile in carotid and/or femoral arteries. The expression of DR3 mRNA but neither TL1A nor DcR3 shows a trend to evaluation in atheromatous plaques of arterial tissue. Collectively, these data highlights that TL1ADR3/DcR3 signaling is involved in chronic inflammation and atherosclerosis.

5.2. Human IBD. In IBD patients, TL1A expression is increased in both serum and intestinal tissues and is correlated with the disease activity [67-69]. DR3 expression is also increased in lymphocytes, in particular $\mathrm{T}$ cells, in the intestinal lamina propria in these patients [68]. Interestingly, TL1A-expressing cells in the lamina propria are macrophages and $\mathrm{CD}^{+}$or $\mathrm{CD}^{+} \mathrm{T}$ cells in $\mathrm{CD}$ patients, whereas they are mainly plasma cells in UC patients [68]. The diversity of TL1A expression might reflect differences in the pathogenesis of CD and UC. TL1A expression is increased only in lamina propria $\mathrm{CD} 14^{+}$macrophages (but not peripheral monocytes or monocyte-derived macrophages) in CD patients but not in UC patients or healthy subjects. The membranebound expression of TL1A is induced in lamina propria $\mathrm{CD} 14^{+}$macrophages stimulated by commensal bacteria such as E. coli [70]. In UC, IgG-producing plasma cells were found to infiltrate areas of mucosal inflammation, and IgG immune complex stimulation increased TL1A expression in macrophages in the lamina propria [71]. These findings indicate that mononuclear phagocytes are likely to be a major source of TL1A in inflamed loci in the intestines of IBD patients. In CD patients, exogenous TL1A and agonistic antiDR3 antibodies augment IFN- $\gamma$ production in lamina propria mononuclear cells as well as peripheral blood mononuclear cells $[68,69]$. TL1A synergizes with IL-12/IL-18 and induces IFN- $\gamma$ production in lamina propria $\mathrm{CCR}^{+} \mathrm{T}$ cells derived from CD patients, which is thought to be implicated in the pathogenesis of CD [72]. Exogenous TL1A and IL-23 synergistically induce IFN- $\gamma$ and IL-17 secretion in lamina propria $\mathrm{CD}^{+}{ }^{+} \mathrm{T}$ cells. TL1A enhances the differentiation of IL-17and IFN- $\gamma /$ IL-17-producing Th17 cells when naïve $\mathrm{CD} 4^{+} \mathrm{T}$ cells are stimulated with lamina propria macrophages derived from CD patients [70]. These reports demonstrate that TL1ADR3 interaction may contribute to Th1- and Th17-mediated responses that are characteristic of $\mathrm{CD}$.

5.3. IBD Mouse Model. TL1A is increased in the intestines of IBD model mice, including dextran-sodium-sulfate (DSS-) induced chronic colitis mice and $\mathrm{TNF}^{\triangle \mathrm{ARE}}$ chronic ileitis mice. Its major source is likely to be dendritic cells in mesenteric lymph nodes and small intestinal lamina propria mononuclear cells $[25,32]$. Mice with constitutive TL1A expression in antigen-presenting cells and $\mathrm{T}$ cells show intestinal inflammation and colonic fibrosis with a high percentage of $\mathrm{T}$ cells that are positive for CCR9 and CCR10, 
both of which are gut-homing chemokines in T cells [34]. As with TL1A, DR3 expression is increased in DSS-induced chronic colitis mice, and its transmembrane splicing variant is increased in correlation with inflammation in chronic ileitis mice, $\mathrm{TNF}^{\triangle \mathrm{ARE}}$ mice, and SAMP1/Yit Fc mice [25, 32]. Administration of blocking anti-TL1A monoclonal antibodies inhibits DSS-induced colonic inflammation in mice [32], and DR3-deficient mice are protected from intestinal inflammation even after colitis induction [27]. In mice with 2,4,6-trinitrobenzenesulfonic acid- (TNBS-) induced colitis, colonic inflammation is inhibited by administration of antiTL1A or anti-DR3 antibodies [27]. These reports demonstrate that TL1A-DR3 interaction plays an important role in the pathogenesis of these chronic intestinal inflammatory conditions in mice. TL1A synergizes with IL-12 or IL-23 and induces both IFN- $\gamma$ and IL-17 secretion in $\mathrm{CD}^{+}{ }^{+} \mathrm{T}$ cells derived from gut-associated lymphoid tissue (GALT) of DSSinduced chronic colitis mice [32]. IL-23 itself or IL-23 in combination with TL1A induces IFN- $\gamma /$ IL-17 double-positive $\mathrm{CD}^{+}{ }^{+} \mathrm{T}$ cells that are known to be colitogenic [32]. Sustained TL1A expression in T cells or dendritic cells promotes goblet cell and Paneth cell hyperplasia in the small intestine in mice [26, 27, 34]. Hyperplasia of goblet cells and Paneth cells is associated with elevated Th2 cytokine production $[73,74]$. In mice with sustained TL1A expression, IL-13 and IL-17 expression is increased in mesenteric lymph node cells, ileum, and $\mathrm{CD}^{+} \mathrm{T}$ cells isolated from the lamina propria. Administration of antagonistic anti-IL13 antibodies but not anti-IL17 antibodies attenuates intestinal inflammation in mice constitutively expressing TL1A [27], suggesting that IL13 plays an important role in the small intestinal inflammation induced by sustained TL1A expression on T cells or dendritic cells. Although agonistic anti-DR3 antibodies and glycosphingolipid enhance the production of Th2 cytokines IL-13 and IL-4 in NKT cells [10], mice with sustained TL1A expression and small intestinal inflammation show decreased NKT cells and increased activated and memory CD $4^{+} \mathrm{T}$ cells, suggesting that in these mice the major source of IL-13 is likely to be activated and memory $T$ cells $[26,27]$. In human, CD is associated with the Th1/Th17 cytokines IL-12 and IL-23, and UC with the Th2 cytokine IL-13 [75]. Taken together, these findings in IBD model mice provide evidence that TL1ADR3 interaction may contribute to both Th1/Th17 and Th2 signaling pathways in human IBD.

5.4. Psoriasis. Bamias et al. reported an association of TL1A and its two receptors with psoriasis [76]. TL1A is mainly expressed in keratinocyte, basal cells, vascular cells, and infiltrating inflammatory cells of psoriatic skin but is rarely expressed in those of normal skin. DR3 and DcR3 are expressed in normal skin and are upregulated in psoriatic skin. The expression levels of TL1A and its two receptors are upregulated in lesional skin as compared to nonlesional skin in psoriasis patients, suggesting that TL1A and its two receptors may be involved in the pathogenesis of psoriasis. Immunohistochemical staining shows that TL1A localizes at nuclear region in inflammatory and fibroblast-like cells in psoriasis patients, although previous studies reported that
TL1A localizes at cytoplasmic region in inflamed tissues of several autoimmune diseases $[6,68]$. In this paper, the unique nuclear location is also confirmed in synoviocytes and inflammatory cells in RA patients. Further study is needed to show the significance of nuclear TL1A localization. TL1A transgenic mice develop chronic intestinal inflammation and a minority of the mice also develop ulcerative skin lesions and arthritis. Concurrent rate among IBD, RA, and psoriasis is high in human. These reports might provide the evidence that TL1A is one of common denominators of gut, joint, and skin inflammation.

5.5. $P B C$. We recently found that TL1A expression is increased in both serum and liver tissues of PBC patients. In the liver, TL1A expression is positive for infiltrating mononuclear cells, endothelial cells, Kupffer cells, and biliary epithelial cells. Serum TL1A levels are decreased in early-stage but not late-stage patients being treated with ursodeoxycholic acid, the only therapeutic drug for PBC approved by the Food and Drug Administration, suggesting that TL1A has a potential to be a new serum marker and therapeutic target for PBC [77].

\section{The Association of DcR3 with Autoimmune and Inflammatory Diseases}

DcR3, also known as TR6, and M68, is a member of the TNFRSF. DcR3 consists of 300 amino acids lacking a transmembrane domain of TNFRSF and be released as secreted protein. DcR3 functions as a decoy receptor for TL1A as well as FasL and LIGHT and inhibits these ligands mediated apoptosis and lymphokine secretion [78-81]. DcR3 is induced in human antigen-presenting cells such as monocytes and myeloid dendritic cells and intestinal epithelial cells lines by lipopolysaccharide or lipoteichoic acid and is also induced in human dermal microvascular endothelial cells by TNF- $\alpha$ and IL-1 $\beta[82,83]$. DcR3 modulates the differentiation and maturation of monocyte, macrophage, and dendritic cells, polarization of naïve T cells into Th-2 immune response [84], and the negative regulation for activation of $\mathrm{B}$ cells by TLR ligands [85]. DcR3 is rarely detectable in serum of healthy subjects, whereas its expression is increased in that of various autoimmune and inflammatory diseases such as IBD $[67,86]$, SLE [78], RA [60], PBC [77], silicosis [87], viral infections [82], renal failure [88], and atopic dermatitis [89] as well as cancer [90]. DcR3 is not found in mouse genome, suggesting that additional complexity of TL1A-DR3 pathway in human as compared to mouse. DcR3 protects the development of autoimmune diabetes [91, 92], IgA nephropathy [93], and crescent glomerulonephritis [94] model mice, while DcR3transgenic mice develop SLE-like syndrome [95]. These reports suggest that DcR3 also plays an important role in the pathogenesis of autoimmune and inflammatory diseases.

\section{Conclusion}

TL1A expression is transiently induced by inflammatory cytokines, TLR ligands, enteric bacteria, and $\mathrm{Fc} \gamma \mathrm{R}$ 


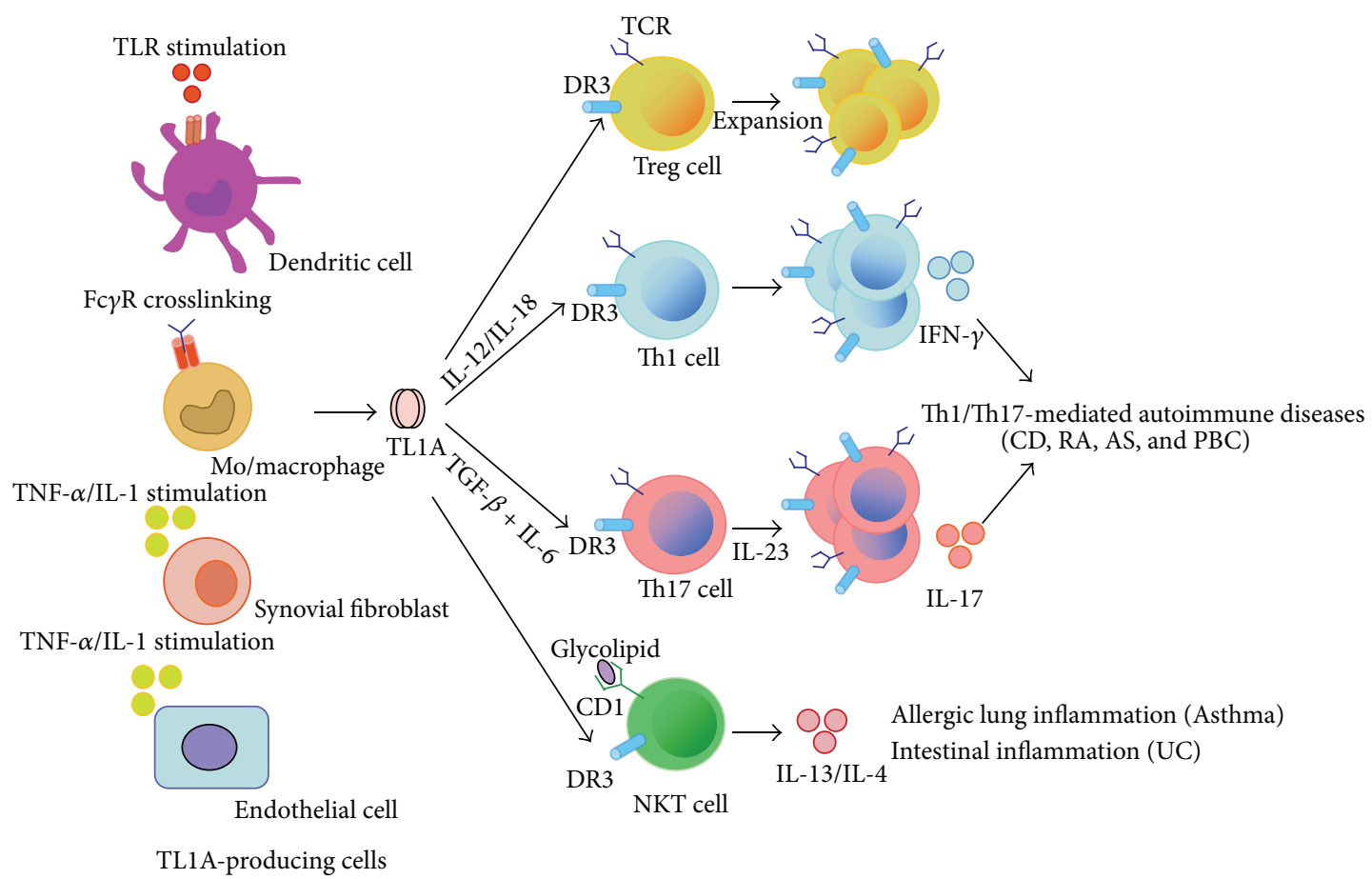

FIGURE 1: TL1A connects innate immune responses to adaptive immune responses and is critically involved in the induction of autoimmune and inflammatory diseases.

crosslinking in antigen-presenting cells or non-immune cells. Although TL1A was initially characterized as a costimulator for inducing cell proliferation and cytokine secretion in $\mathrm{T}$ cells, there is growing evidence that TL1A has pleiotropic effects such as cell death, differentiation, and maintenance of lymphocytes, as well as osteoclastogenesis and atherosclerosis. Increased TL1A expression and/or TL1A (TNFSF15) gene polymorphisms are associated with the pathogenesis of various autoimmune and inflammatory diseases. Analysis of murine models of autoimmune diseases and TL1A or DR3 transgenic mice suggests that TL1A-DR3 interaction plays an important role in local inflammation of T-cell-dependent autoimmune diseases. Thus, TL1A connects innate immune responses to adaptive immune responses and is critically involved in the induction of autoimmune and inflammatory diseases (Figure 1), suggesting that inhibition of TL1A-DR3 interaction could be an effective therapeutic strategy for ameliorating local inflammation in target organs of individuals with autoimmune diseases.

\section{Conflict of Interests}

The authors declare that they have no conflict of interests.

\section{Acknowledgments}

This work was supported by a Grant-in-Aid for Scientific Research from the Japan Society for the Promotion of Science to Yoshihiro Aiba (no. 25860576) and Minoru Nakamura (no. 23591006); by a Grant-in-Aid for Clinical Research from the
National Hospital Organization to Minoru Nakamura; and by a grant from the Research Program of Intractable Disease provided by the Ministry of Health, Labour and Welfare of Japan.

\section{References}

[1] T.-S. Migone, J. Zhang, X. Luo et al., "TL1A is a TNF-like ligand for DR3 and TR6/DcR3 and functions as a T cell costimulator," Immunity, vol. 16, no. 3, pp. 479-492, 2002.

[2] C. Zhan, Q. Yan, Y. Patskovsky et al., "Biochemical and structural characterization of the human TL1A ectodomain," Biochemistry, vol. 48, no. 32, pp. 7636-7645, 2009.

[3] C. Mück, D. Herndler-Brandstetter, L. Micutkova, B. GrubeckLoebenstein, and P. Jansen-Dürr, "Two functionally distinct isoforms of TL1A (TNFSF15) generated by differential ectodomain shedding," The Journals of Gerontology A, vol. 65, no. 11, pp. 1165-1180, 2010.

[4] J. Zhang, X. Wang, H. Fahmi et al., "Role of TL1A in the pathogenesis of rheumatoid arthritis," Journal of Immunology, vol. 183, no. 8, pp. 5350-5357, 2009.

[5] J. L. Prehn, L. S. Thomas, C. J. Landers, Q. T. Yu, K. S. Michelsen, and S. R. Targan, "The T cell costimulator TL1A is induced by $\mathrm{Fc} \gamma \mathrm{R}$ signaling in human monocytes and dendritic cells," Journal of Immunology, vol. 178, no. 7, pp. 4033-4038, 2007.

[6] M. A. Cassatella, G. P. da Silva, I. Tinazzi et al., "Soluble TNF-like cytokine (TL1A) production by immune complexes stimulated monocytes in rheumatoid arthritis," Journal of Immunology, vol. 178, no. 11, pp. 7325-7333, 2007.

[7] D. Q. Shih, L. Y. Kwan, V. Chavez et al., "Microbial induction of inflammatory bowel disease associated gene TL1A (TNFSF15) 
in antigen presenting cells," European Journal of Immunology, vol. 39, no. 11, pp. 3239-3250, 2009.

[8] G. R. Screaton, X.-N. Xu, A. L. Olsen et al., "LARD: a new lymphoid-specific death domain containing receptor regulated by alternative pre-mRNA splicing," Proceedings of the National Academy of Sciences of the United States of America, vol. 94, no. 9, pp. 4615-4619, 1997.

[9] K. B. Tan, J. Harrop, M. Reddy et al., "Characterization of a novel TNF-like ligand and recently described TNF ligand and TNF receptor superfamily genes and their constitutive and inducible expression in hematopoietic and non-hematopoietic cells," Gene, vol. 204, no. 1-2, pp. 35-46, 1997.

[10] L. Fang, B. Adkins, V. Deyev, and E. R. Podack, "Essential role of TNF receptor superfamily 25 (TNFRSF25) in the development of allergic lung inflammation," The Journal of Experimental Medicine, vol. 205, no. 5, pp. 1037-1048, 2008.

[11] G. W. Jones, J. S. Stumhofer, T. Foster et al., "Naive and activated $\mathrm{T}$ cells display differential responsiveness to TL1A that affects Th17 generation, maintenance, and proliferation," The Journal of the Federation of American Societies for Experimental Biology, vol. 25, no. 1, pp. 409-419, 2011.

[12] T. H. Schreiber, D. Wolf, M. S. Tsai et al., "Therapeutic Treg expansion in mice by TNFRSF25 prevents allergic lung inflammation," The Journal of Clinical Investigation, vol. 120, no. 10, pp. 3629-3640, 2010.

[13] B. P. Pappu, A. Borodovsky, T. S. Zheng et al., "TL1A-DR3 interaction regulates Thl7 cell function and Thl7-mediated autoimmune disease," The Journal of Experimental Medicine, vol. 205, no. 5, pp. 1049-1062, 2008.

[14] C. Cavallini, O. Lovato, A. Bertolaso et al., "The TNF-family cytokine TL1A inhibits proliferation of human activated B cells," PloS One, vol. 8, no. 4, Article ID e60136, 2013.

[15] J. Kitson, T. Raven, Y.-P. Jiang et al., "A death-domaincontaining receptor that mediates apoptosis," Nature, vol. 384, no. 6607 , pp. 372-375, 1996.

[16] K. Warzocha, P. Ribeiro, C. Charlot, N. Renard, B. Coiffier, and G. Salles, "New death receptor 3 isoform: expression in human lymphoid cell lines and non-Hodgkin's lymphomas," Biochemical and Biophysical Research Communications, vol. 242, no. 2, pp. 376-379, 1998.

[17] E. C. Y. Wang, J. Kitson, A. Thern, J. Williamson, S. N. Farrow, and M. J. Owen, "Genomic structure, expression, and chromosome mapping of the mouse homologue for the WSL-1 (DR3, Apo3, TRAMP, LARD, TR3, TNFRSF12) gene," Immunogenetics, vol. 53, no. 1, pp. 59-63, 2001.

[18] J.-L. Bodmer, K. Burns, P. Schneider et al., “TRAMP, a novel apoptosis-mediating receptor with sequence homology to tumor necrosis factor receptor 1 and Fas(Apo-1/CD95)," Immunity, vol. 6, no. 1, pp. 79-88, 1997.

[19] A. M. Chinnaiyan, K. O’Rourke, G.-L. Yu et al., "Signal transduction by DR3, a death domain-containing receptor related to TNFR-1 and CD95," Science, vol. 274, no. 5289, pp. 990-992, 1996.

[20] L. Wen, L. Zhuang, X. Luo, and P. Wei, “TL1A-induced NF$\kappa \mathrm{B}$ activation and c-IAP2 production prevent DR3-mediated apoptosis in TF-1 cells," The Journal of Biological Chemistry, vol. 278, no. 40, pp. 39251-39258, 2003.

[21] E. E. Varfolomeev, M. Schuchmann, V. Luria et al., "Targeted disruption of the mouse Caspase 8 gene ablates cell death induction by the TNF receptors, Fas/Apol, and DR3 and is lethal prenatally," Immunity, vol. 9, no. 2, pp. 267-276, 1998.
[22] W.-C. Yeh, J. L. de la Pompa, M. E. McCurrach et al., "FADD: essential for embryo development and signaling from some, but not all, inducers of apoptosis," Science, vol. 279, no. 5358, pp. 1954-1958, 1998.

[23] E. C. Y. Wang, A. Thern, A. Denzel, J. Kitson, S. N. Farrow, and M. J. Owen, "DR3 regulates negative selection during thymocyte development," Molecular and Cellular Biology, vol. 21, no. 10, pp. 3451-3461, 2001.

[24] F. Meylan, T. S. Davidson, E. Kahle et al., "The TNF-family receptor DR3 is essential for diverse T cell-mediated inflammatory diseases," Immunity, vol. 29, no. 1, pp. 79-89, 2008.

[25] G. Bamias, M. Mishina, M. Nyce et al., "Role of TL1A and its receptor DR3 in two models of chronic murine ileitis," Proceedings of the National Academy of Sciences of the United States of America, vol. 103, no. 22, pp. 8441-8446, 2006.

[26] V. Y. Taraban, T. J. Slebioda, J. E. Willoughby et al., "Sustained TL1A expression modulates effector and regulatory T-cell responses and drives intestinal goblet cell hyperplasia," Mucosal Immunology, vol. 4, no. 2, pp. 186-196, 2011.

[27] F. Meylan, Y.-J. Song, I. Fuss et al., "The TNF-family cytokine TL1A drives IL-13-dependent small intestinal inflammation," Mucosal Immunology, vol. 4, no. 2, pp. 172-185, 2011.

[28] K. A. Papadakis, J. L. Prehn, C. Landers et al., "TL1A synergizes with IL-12 and IL-18 to enhance IFN- $\gamma$ production in human T cells and NK cells," Journal of Immunology, vol. 172, no. 11, pp. 7002-7007, 2004.

[29] J. P. Twohig, M. Marsden, S. M. Cuff et al., "The death receptor 3/TL1A pathway is essential for efficient development of antiviral CD4(+) and CD8(+) T-cell immunity," The Journal of the Federation of American Societies For Experimental Biology, vol. 26, no. 8, pp. 3575-3586, 2012.

[30] A. Laurence, C. M. Tato, T. S. Davidson et al., "Interleukin2 signaling via STAT5 constrains T helper 17 cell generation," Immunity, vol. 26, no. 3, pp. 371-381, 2007.

[31] L. Liu, S. Okada, X. F. Kong et al., "Gain-of-function human STAT1 mutations impair IL-17 immunity and underlie chronic mucocutaneous candidiasis," The Journal of Experimental Medicine, vol. 208, no. 8, pp. 1635-1648, 2011.

[32] H. Takedatsu, K. S. Michelsen, B. Wei et al., "TL1A (TNFSF15) regulates the development of chronic colitis by modulating both T-helper 1 and T-helper 17 activation," Gastroenterology, vol. 135, no. 2, pp. 552.e2-567.e2, 2008.

[33] H. Kayamuro, Y. Yoshioka, Y. Abe et al., "TNF superfamily member, TL1A, is a potential mucosal vaccine adjuvant," Biochemical and Biophysical Research Communications, vol. 384, no. 3, pp. 296-300, 2009.

[34] D. Q. Shih, R. Barrett, X. Zhang et al., "Constitutive TL1A (TNFSF15) expression on lymphoid or myeloid cells leads to mild intestinal inflammation and fibrosis," PLoS ONE, vol. 6, no. 1, Article ID e16090, 2011.

[35] S. Q. Khan, M. S. Tsai, T. H. Schreiber et al., "Cloning, expression, and functional characterization of TL1A-Ig," Journal of Immunology, vol. 190, no. 4, pp. 1540-1550, 2013.

[36] S. C. Heidemann, V. Chavez, C. J. Landers, T. Kucharzik, J. L. Prehn, and S. R. Targan, "TL1A selectively enhances IL-12/IL18-induced NK cell cytotoxicity against NK-resistant tumor targets," Journal of Clinical Immunology, vol. 30, no. 4, pp. 531538, 2010.

[37] K. Yamazaki, D. McGovern, J. Ragoussis et al., "Single nucleotide polymorphisms in TNFSF15 confer susceptibility to Crohn's disease," Human Molecular Genetics, vol. 14, no. 22, pp. 3499-3506, 2005. 
[38] M. Tremelling, C. Berzuini, D. Massey et al., "Contribution of TNFSF15 gene variants to Crohn's disease susceptibility confirmed in UK population," Inflammatory Bowel Diseases, vol. 14, no. 6, pp. 733-737, 2008.

[39] Y. Kakuta, Y. Kinouchi, K. Negoro, S. Takahashi, and T. Shimosegawa, "Association study of TNFSF15 polymorphisms in Japanese patients with inflammatory bowel disease," Gut, vol. 55, no. 10, pp. 1527-1528, 2006.

[40] J. C. Barrett, S. Hansoul, D. L. Nicolae et al., "Genome-wide association defines more than 30 distinct susceptibility loci for Crohn's disease," Nature Genetics, vol. 40, no. 8, pp. 955-962, 2008.

[41] A. Franke, T. Balschun, T. H. Karlsen et al., "Replication of signals from recent studies of Crohn's disease identifies previously unknown disease loci for ulcerative colitis," Nature Genetics, vol. 40, no. 6, pp. 713-715, 2008.

[42] K. Wang, R. Baldassano, H. Zhang et al., "Comparative genetic analysis of inflammatory bowel disease and type 1 diabetes implicates multiple loci with opposite effects," Human Molecular Genetics, vol. 19, no. 10, pp. 2059-2067, 2010.

[43] R. Thiébaut, S. Kotti, C. Jung et al., “TNFSF15 polymorphisms are associated with susceptibility to inflammatory bowel disease in a new European cohort," The American Journal of Gastroenterology, vol. 104, no. 2, pp. 384-391, 2009.

[44] S.-K. Yang, J. Lim, H.-S. Chang et al., "Association of TNFSF15 with Crohn's disease in Koreans," The American Journal of Gastroenterology, vol. 103, no. 6, pp. 1437-1442, 2008.

[45] L. Jostins, S. Ripke, R. K. Weersma et al., "Host-microbe interactions have shaped the genetic architecture of inflammatory bowel disease," Nature, vol. 491, no. 7422, pp. 119-124, 2012.

[46] A. Hirano, K. Yamazaki, J. Umeno et al., "Association study of 71 European Crohn's disease susceptibility loci in a Japanese population," Inflammatory Bowel Diseases, vol. 19, no. 3, pp. 526-533, 2013.

[47] I. Cleynen, J. R. Gonzalez, C. Figueroa et al., "Genetic factors conferring an increased susceptibility to develop Crohn's disease also influence disease phenotype: results from the IBDchip European Project," Gut, vol. 62, no. 11, pp. 1556-1565, 2012.

[48] T. Haritunians, K. D. Taylor, S. R. Targan et al., "Genetic predictors of medically refractory ulcerative colitis," Inflammatory Bowel Diseases, vol. 16, no. 11, pp. 1830-1840, 2010.

[49] Y. Picornell, L. Mei, K. Taylor, H. Yang, S. R. Targan, and J. I. Rotter, "TNFSF15 is an ethnic-specific IBD gene," Inflammatory Bowel Diseases, vol. 13, no. 11, pp. 1333-1338, 2007.

[50] K. S. Michelsen, L. S. Thomas, K. D. Taylor et al., "IBDassociated TL1A gene (TNFSFI5) haplotypes determine increased expression of TL1A protein," PLOS ONE, vol. 4, no. 3, Article ID e4719, 2009.

[51] Y. Kakuta, N. Ueki, Y. Kinouchi et al., "TNFSF15 transcripts from risk haplotype for Crohn's disease are overexpressed in stimulated T cells," Human Molecular Genetics, vol. 18, no. 6, pp. 1089-1098, 2009.

[52] M. Zucchelli, M. Camilleri, A. N. Andreasson et al., "Association of TNFSF15 polymorphism with irritable bowel syndrome," Gut, vol. 60, no. 12, pp. 1671-1677, 2011.

[53] E. Zinovieva, C. Bourgain, A. Kadi et al., "Comprehensive linkage and association analyses identify haplotype, near to the TNFSF15 gene, significantly associated with spondyloarthritis," PLoS Genetics, vol. 5, no. 6, Article ID e1000528, 2009.

[54] M. Nakamura, N. Nishida, M. Kawashima et al., "Genomewide association study identifies TNFSF15 and POU2AF1 as susceptibility loci for primary biliary cirrhosis in the Japanese population," American Journal of Human Genetics, vol. 91, no. 4, pp. 721-728, 2012.

[55] A. Franke, D. P. McGovern, J. C. Barrett et al., "Genome-wide meta-analysis increases to 71 the number of confirmed Crohn's disease susceptibility loci," Nature Genetics, vol. 42, no. 12, pp. 1118-1125, 2010.

[56] C. A. Anderson, G. Boucher, C. W. Lees et al., "Meta-analysis identifies 29 additional ulcerative colitis risk loci, increasing the number of confirmed associations to 47," Nature Genetics, vol. 43, no. 3, pp. 246-252, 2011.

[57] B. D. Juran and K. N. Lazaridis, "Update on the genetics and genomics of PBC," Journal of Autoimmunity, vol. 35, no. 3, pp. 181-187, 2010.

[58] F. R. Zhang, W. Huang, S. M. Chen et al., "Genomewide association study of leprosy," The New England Journal of Medicine, vol. 361, no. 27, pp. 2609-2618, 2009.

[59] F. Zhang, H. Liu, S. Chen et al., "Identification of two new loci at IL23R and RAB32 that influence susceptibility to leprosy," Nature Genetics, vol. 43, no. 12, pp. 1247-1251, 2011.

[60] G. Bamias, S. I. Siakavellas, K. S. Stamatelopoulos, E. Chryssochoou, C. Papamichael, and P. P. Sfikakis, "Circulating levels of TNF-like cytokine 1A (TL1A) and its decoy receptor 3 (DcR3) in rheumatoid arthritis," Clinical Immunology, vol. 129, no. 2, pp. 249-255, 2008.

[61] K. Osawa, N. Takami, K. Shiozawa, A. Hashiramoto, and S. Shiozawa, "Death receptor 3 (DR3) gene duplication in a chromosome region 1p36.3: gene duplication is more prevalent in rheumatoid arthritis," Genes and Immunity, vol. 5, no. 6, pp. 439-443, 2004.

[62] Y.-J. Kang, W.-J. Kim, H.-U. Bae et al., "Involvement of TL1A and DR3 in induction of pro-inflammatory cytokines and matrix metalloproteinase-9 in atherogenesis," Cytokine, vol. 29, no. 5, pp. 229-235, 2005.

[63] M. J. Bull, A. S. Williams, Z. Mecklenburgh et al., "The death receptor 3-TNF-like protein $1 \mathrm{~A}$ pathway drives adverse bone pathology in inflammatory arthritis," The Journal of Experimental Medicine, vol. 205, no. 11, pp. 2457-2464, 2008.

[64] D. H. Solomon, N. J. Goodson, J. N. Katz et al., "Patterns of cardiovascular risk in rheumatoid arthritis," Annals of the Rheumatic Diseases, vol. 65, no. 12, pp. 1608-1612, 2006.

[65] J. E. McLaren, C. J. Calder, B. P. McSharry et al., "The TNFlike protein $1 \mathrm{~A}$-death receptor 3 pathway promotes macrophage foam cell formation in vitro," Journal of Immunology, vol. 184, no. 10, pp. 5827-5834, 2010.

[66] G. Bamias, K. Stamatelopoulos, E. Zampeli et al., "Circulating levels of TNF-like cytokine 1A correlate with the progression of atheromatous lesions in patients with rheumatoid arthritis," Clinical Immunology, vol. 147, no. 2, pp. 144-150, 2013.

[67] G. Bamias, G. Kaltsa, S. I. Siakavellas et al., "High intestinal and systemic levels of decoy receptor 3 (DcR3) and its ligand TL1A in active ulcerative colitis," Clinical Immunology, vol. 137, no. 2, pp. 242-249, 2010.

[68] G. Bamias, C. Martin III, M. Marini et al., "Expression, localization, and functional activity of TL1A, a novel Th1polarizing cytokine in inflammatory bowel disease," Journal of Immunology, vol. 171, no. 9, pp. 4868-4874, 2003.

[69] J. L. Prehn, S. Mehdizadeh, C. J. Landers et al., "Potential role for TL1A, the new TNF-family member and potent costimulator of IFN- $\gamma$, in mucosal inflammation," Clinical Immunology, vol. 112, no. 1, pp. 66-77, 2004. 
[70] N. Kamada, T. Hisamatsu, H. Honda et al., "TL1A produced by lamina propria macrophages induces Thl and Th17 immune responses in cooperation with IL-23 in patients with Crohn's disease," Inflammatory Bowel Diseases, vol. 16, no. 4, pp. 568$575,2010$.

[71] M. Uo, T. Hisamatsu, J. Miyoshi et al., "Mucosal CXCR4+ IgG plasma cells contribute to the pathogenesis of human ulcerative colitis through FcgammaR-mediated CD14 macrophage activation," Gut, vol. 62, no. 12, pp. 1734-1744, 2013.

[72] M. Saruta, Q. T. Yu, A. Avanesyan, P. R. Fleshner, S. R. Targan, and K. A. Papadakis, "Phenotype and effector function of CC chemokine receptor 9-expressing lymphocytes in small intestinal Crohn's disease," Journal of Immunology, vol. 178, no. 5, pp. 3293-3300, 2007.

[73] V. Steenwinckel, J. Louahed, M. M. Lemaire et al., "IL-9 promotes IL-13-dependent paneth cell hyperplasia and upregulation of innate immunity mediators in intestinal mucosa," Journal of Immunology, vol. 182, no. 8, pp. 4737-4743, 2009.

[74] Y. S. Kim and S. B. Ho, "Intestinal goblet cells and mucins in health and disease: recent insights and progress," Current Gastroenterology Reports, vol. 12, no. 5, pp. 319-330, 2010.

[75] I. J. Fuss, "Is the Th1/Th2 paradigm of immune regulation applicable to IBD?" Inflammatory Bowel Diseases, vol. 14, supplement 2, pp. S110-S112, 2008.

[76] G. Bamias, K. Evangelou, T. Vergou et al., "Upregulation and nuclear localization of TNF-like Cytokine 1A (TL1A) and its receptors DR3 and DcR3 in psoriatic skin lesions," Experimental Dermatology, vol. 20, no. 9, pp. 725-731, 2011.

[77] Y. Aiba, K. Harada, A. Komori et al., "Systemic and local expression levels of TNF-like ligand 1A and its decoy receptor 3 are increased in primary biliary cirrhosis," Liver International, 2013.

[78] C.-S. Lee, C.-Y. Hu, H.-F. Tsai et al., "Elevated serum decoy receptor 3 with enhanced $\mathrm{T}$ cell activation in systemic lupus erythematosus," Clinical and Experimental Immunology, vol. 151, no. 3, pp. 383-390, 2008.

[79] J. Zhang, T. W. Salcedo, X. Wan et al., "Modulation of T-cell responses to alloantigens by TR6/DcR3," The Journal of Clinical Investigation, vol. 107, no. 11, pp. 1459-1468, 2001.

[80] K.-Y. Yu, B. Kwon, J. Ni, Y. Zhai, R. Ebner, and B. S. Kwon, "A newly identified member of tumor necrosis factor receptor superfamily (TR6) suppresses LIGHT-mediated apoptosis," The Journal of Biological Chemistry, vol. 274, no. 20, pp. 13733-13736, 1999.

[81] R. M. Pitti, S. A. Marsters, D. A. Lawrence et al., "Genomic amplification of a decoy receptor for Fas ligand in lung and colon cancer," Nature, vol. 396, no. 6712, pp. 699-703, 1998.

[82] S. Kim, W. J. McAuliffe, L. S. Zaritskaya, P. A. Moore, L. Zhang, and B. Nardelli, "Selective induction of tumor necrosis receptor factor $6 /$ decoy receptor 3 release by bacterial antigens in human monocytes and myeloid dendritic cells," Infection and Immunity, vol. 72, no. 1, pp. 89-93, 2004.

[83] S. Kim, A. Fotiadu, and V. Kotoula, "Increased expression of soluble decoy receptor 3 in acutely inflamed intestinal epithelia," Clinical Immunology, vol. 115, no. 3, pp. 286-294, 2005.

[84] T.-L. Hsu, Y.-C. Chang, S.-J. Chen et al., "Modulation of dendritic cell differentiation and maturation by decoy receptor 3," Journal of Immunology, vol. 168, no. 10, pp. 4846-4853, 2002.

[85] Z. M. Huang, J. K. Kang, C. Y. Chen et al., "Decoy receptor 3 suppresses TLR2-mediated B cell activation by targeting NFkappaB," Journal of Immunology, vol. 188, no. 12, pp. 5867-5876, 2012.
[86] B. Funke, F. Autschbach, S. Kim et al., "Functional characterisation of decoy receptor 3 in Crohn's disease," Gut, vol. 58, no. 4, pp. 483-491, 2009.

[87] T. Otsuki, A. Tomokuni, H. Sakaguchi et al., "Over-expression of the decoy receptor 3 (DcR3) gene in peripheral blood mononuclear cells (PBMC) derived from silicosis patients," Clinical and Experimental Immunology, vol. 119, no. 2, pp. 323327, 2000.

[88] J. Chen, L. Zhang, and S. Kim, "Quantification and detection of DcR3, a decoy receptor in TNFR family," Journal of Immunological Methods, vol. 285, no. 1, pp. 63-70, 2004.

[89] C. Chun Chen, Y. Hsu Yang, Y. Tsan Lin, S. Liang Hsieh, and B.L. Chiang, "Soluble decoy receptor 3: increased levels in atopic patients," The Journal of Allergy and Clinical Immunology, vol. 114, no. 1, pp. 195-197, 2004.

[90] Y. Wu, B. Han, H. Sheng et al., "Clinical significance of detecting elevated serum DcR3/TR6/M68 in malignant tumor patients," International Journal of Cancer, vol. 105, no. 5, pp. 724-732, 2003.

[91] Y.-L. Wang, F.-C. Chou, H.-H. Sung et al., "Decoy receptor 3 protects non-obese diabetic mice from autoimmune diabetes by regulating dendritic cell maturation and function," Molecular Immunology, vol. 47, no. 16, pp. 2552-2562, 2010.

[92] H.-H. Sung, J.-H. Juang, Y.-C. Lin et al., "Transgenic expression of decoy receptor 3 protects islets from spontaneous and chemical-induced autoimmune destruction in nonobese diabetic mice," The Journal of Experimental Medicine, vol. 199, no. 8, pp. 1143-1151, 2004.

[93] S.-M. Ka, T.-T. Hsieh, S.-H. Lin et al., "Decoy receptor 3 inhibits renal mononuclear leukocyte infiltration and apoptosis and prevents progression of IgA nephropathy in mice," American Journal of Physiology-Renal Physiology, vol. 301, no. 6, pp. F1218-F1230, 2011.

[94] S.-M. Ka, H.-K. Sytwu, D.-M. Chang, S.-L. Hsieh, P.-Y. Tsai, and A. Chen, "Decoy receptor 3 ameliorates an autoimmune crescentic glomerulonephritis model in mice," Journal of the American Society of Nephrology, vol. 18, no. 9, pp. 2473-2485, 2007.

[95] B. Han, P. A. Moore, J. Wu, and H. Luo, "Overexpression of human decoy receptor 3 in mice results in a systemic lupus erythematosus-like syndrome," Arthritis and Rheumatism, vol. 56, no. 11, pp. 3748-3758, 2007. 


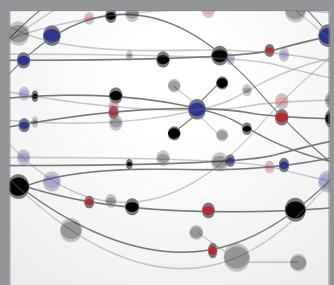

The Scientific World Journal
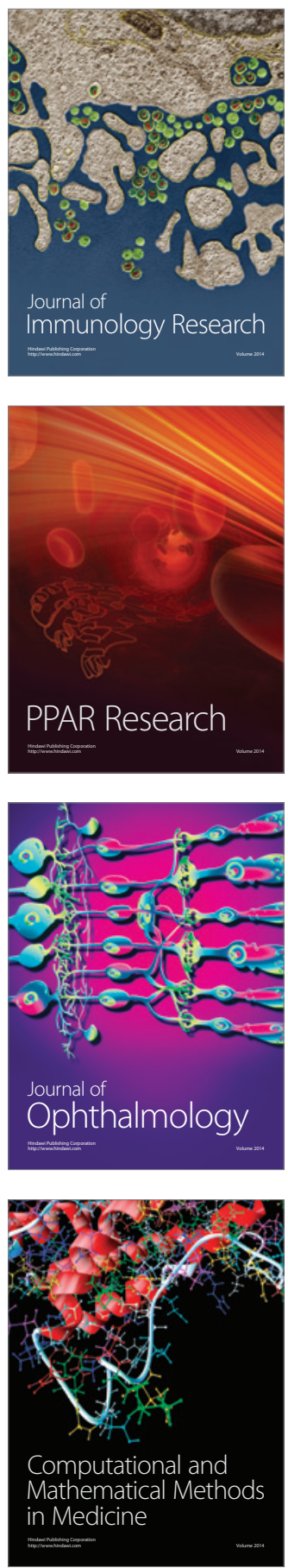

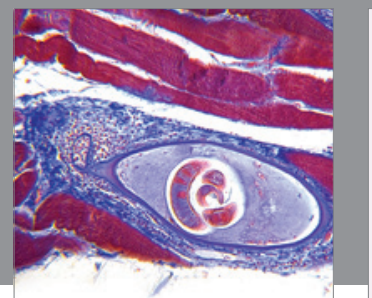

Gastroenterology

Research and Practice
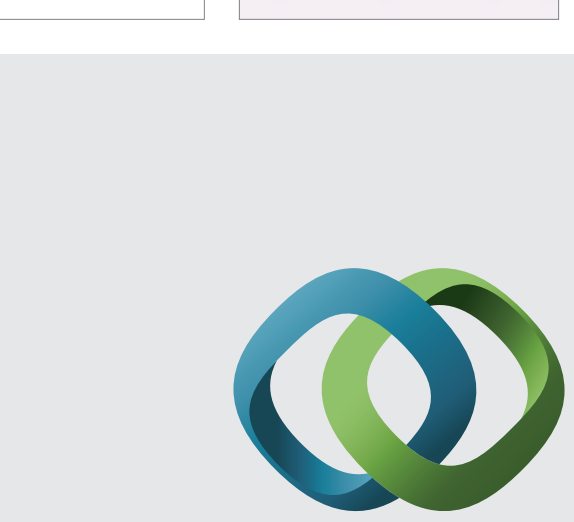

\section{Hindawi}

Submit your manuscripts at

http://www.hindawi.com
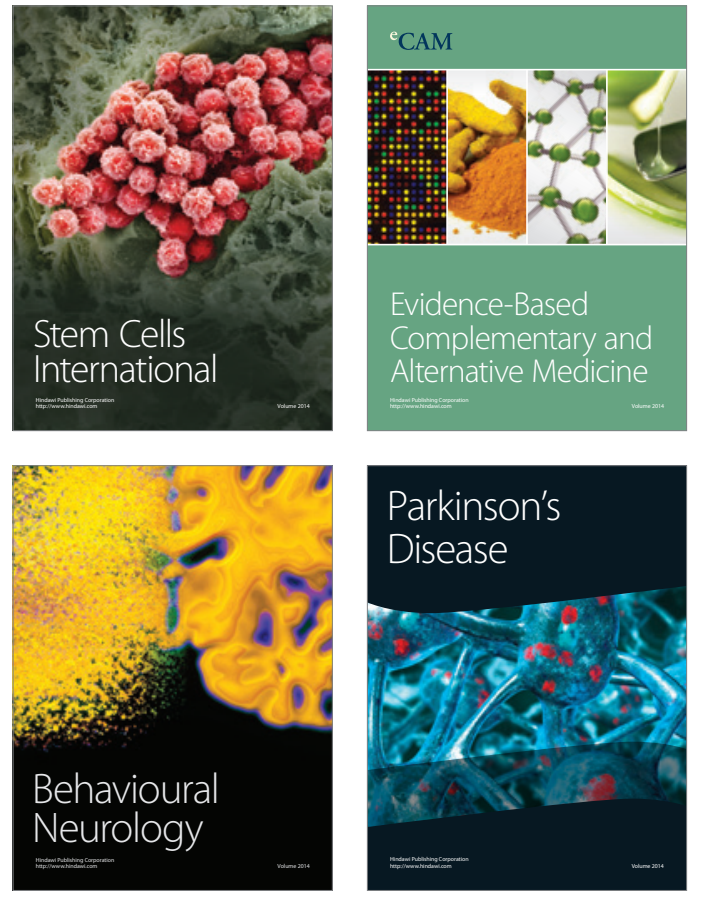
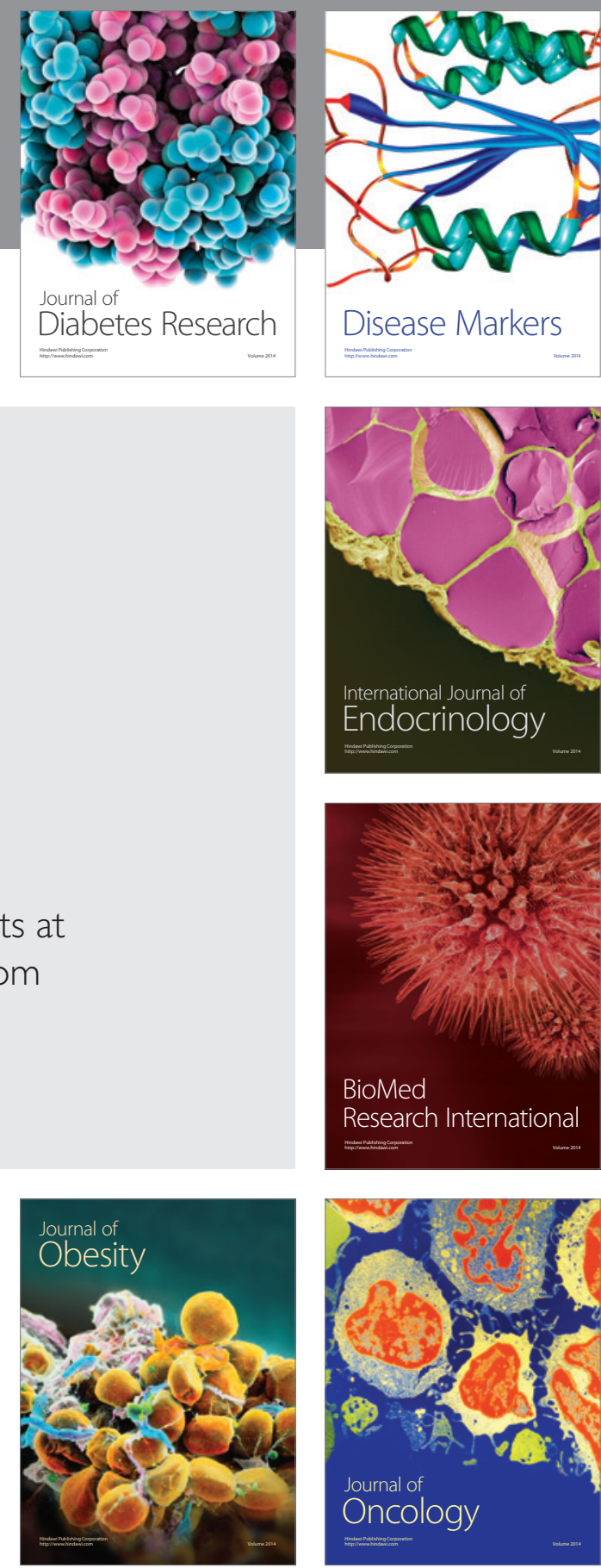

Disease Markers
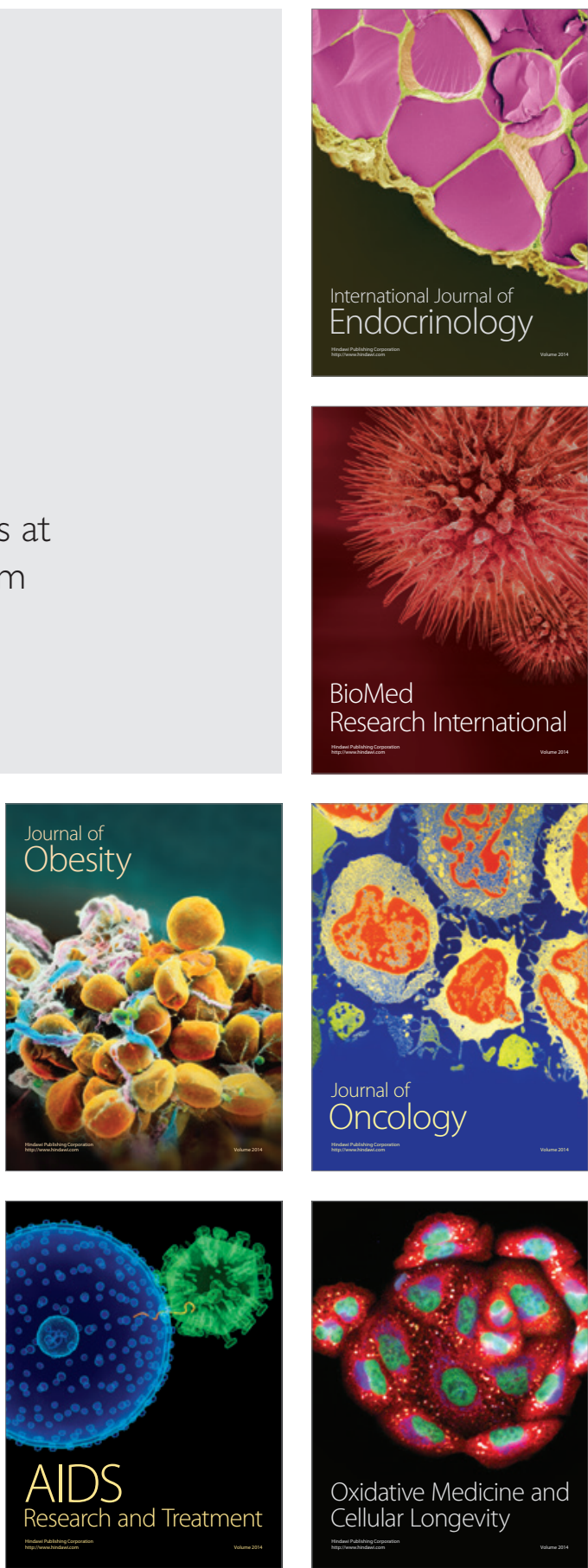